\title{
ON THE SEMICLASSICAL TREATMENT OF HOT NUCLEAR SYSTEMS 尔
}

\author{
J. BARTEL \\ Laboratoire de Physique Théorique, Université de Bordeaux I, F-33170 Gradignan, France \\ and Institut für Theoretische Physik, Universität Regensburg, D-8400 Regensburg, West Germany ${ }^{1}$ \\ M. BRACK ${ }^{2}$, C. GUET \\ DRF-CPN, Centre d'Etudes Nucléaires Grenoble (CENG), F-38041 Grenoble, France \\ and \\ H.-B. HÅKANSSON ${ }^{3}$ \\ Institut für Theoretische Physik, Universität Regensburg, D-8400 Regensburg, West Germany
}

Received 21 December 1983

\begin{abstract}
We discuss two different semiclassical approaches for calculating properties of hot nuclei and compare them to Hartree-Fock calculations using the same effective interaction. Good agreement is found for the entropy and the root-mean square radii as functions of the excitation energy. For a realistic Skyrme force we evaluate the temperature dependence of the free surface, curvature and constant energy coefficients of the liquid drop model, considering a plane interface of condensed symmetric nuclear matter in thermodynamical equilibrium with a nucleon gas.
\end{abstract}

Hot nuclear systems are of actual interest both in heavy-ion physics and in astrophysics. Nuclear compound systems with temperatures of $\sim 3-4 \mathrm{MeV}$ or more can be experimentally produced in heavy-ion or high energy hadron induced reactions [1], and the possibility of measuring the fission barrier of such an excited nucleus is an interesting speculation. In astrophysics, the properties of hot nuclear matter are discussed in the context of the evolution of supernovae [2]. In both applications, simple estimates are most easily made in terms of a liquid drop model (LDM) type mass formula. The temperature dependence of the LDM parameters, in particular the surface and curvature energies, can then play a rather crucial role

Work partially supported by Deutsche Forschungsgemeinschaft.

1 Present address.

2 Permanent address: Institut für Theoretische Physik, Universität Regensburg, D-8400 Regensburg, West Germany.

${ }^{3}$ Present address: ASEA-PFBC AB, S-61220 Finspong, Sweden.
[3]. These parameters can in principle be derived from effective nucleon-nucleon interactions such as used in Hartree-Fock (HF) calculations. However, due to the shell effects, the determination of LDM parameters with purely microscopical methods is rather difficult; for the curvature energy or higher order terms, it may even lead to ambiguous results [4]. A more appropriate and, in fact, also more convenient way is to use semiclassical methods which systematically ignore the shell effects. Such methods are in particular well suited at temperatures above $\sim 1.5-3 \mathrm{MeV}$, where the shell effects are washed out and the systems become semiclassical in nature, so that microscopical calculations entail unnecessary complications.

In the present note we shall discuss two different semiclassical approaches to describe hot nuclear systems. One is the extended Thomas-Fermi (ETF) density functional method [5-7], the other is the socalled resummation method $[8-10]$.

In the ETF model at zero temperature one uses 
the gradient expansion of the kinetic energy density functional $[5,6]$

$\tau_{\mathrm{ETF}}^{T=0}[\rho]=\tau_{\mathrm{TF}}[\rho]+\tau_{2}[\rho]+\tau_{4}[\rho]$,

where $\rho(r)$ is the density of one kind of nucleon. For the case of a local average potential, the functional eq. (1) is universal; the first two terms are the familiar TF term and the Weizsäcker correction, and $\tau_{4}[\rho]$ contains 7 terms with up to fourth derivatives of $\rho$. This functional was shown [11] to reproduce very accurately the average kinetic energy of a given number of nucleons in terms of their average density. Effects of a spin-orbit potential and of variable effective nucleon masses, such as they occur in connection with Skyrme forces, can also be treated in the same way [6]. The total energy density $\epsilon(r)$ of a nucleus can then be written as a functional of the nucleon densities $\rho_{\mathrm{p}}(\boldsymbol{r})$ and $\rho_{\mathrm{n}}(r)$ alone and the latter can be determined by variational calculations. This method was successfully used with effective Skyrme interactions to yield average nuclear ground-state properties and fission barriers $[7,12,13]$ in quantitative agreement with selfconsistently Strutinsky-averaged $\mathrm{HF}$ results [14]. Hereby the gradient corrections $\tau_{2}[\rho]$ and $\tau_{4}[\rho]$ were found to be of crucial importance for obtaining the correct surface properties of both energies and density profiles [7].

Unfortunately, the generalization of the ETF model to finite temperatures is not obvious. In the pure TF approximation, the expressions for $\rho(r), \tau(r)$ and the entropy density $\sigma(r)$ are known [15], and the functionals $\tau_{\mathrm{TF}}^{T>0}[\rho], \sigma_{\mathrm{TF}}[\rho]$ valid at a given temperature can be evaluated numerically. They have been used in TF calculations for the thermal properties of both infinite [15] and semi-infinite nuclear matter $[16,17]$. However, the correct $T$-dependence of the gradient corrections to $\tau[\rho]$ and $\sigma[\rho]$ is not known. We shall investigate here the approximate functional obtained by adding to the exact finite-temperature TF relation $\tau_{\mathrm{TF}}^{T>0}[\rho]$ the gradient corrections known from the $T=0$ case, thus defining

$\tau_{\mathrm{ETF}}[\rho]=\tau_{\mathrm{TF}}^{T>0}[\rho]+\left(\tau_{\mathrm{ETF}}^{T=0}[\rho]-\tau_{\mathrm{TF}}^{T=0}[\rho]\right)$.

Although this procedure cannot be formally justified, it appears reasonable since the $T$-dependence is treated exactly in the leading TF term, and eq. (2) has the correct limit for $T=0$. It was proposed also by Barranco and Treiner [18] who, however, use a sim- plified phenomenological functional $\tau^{T=0}[\rho]$. Below we shall always include the full fourth order functional $\tau_{\text {ETF }}^{T=0}[\rho]$ and the corresponding spin - orbit and effective mass contributions given in refs. [6,7]. We shall also consider the approximation obtained if the TF functionals are treated in the low temperature expansion, valid if $T \ll(\lambda-V)$, where $V$ is the local potential. In this limit one finds

$$
\begin{aligned}
& \tau_{\mathrm{ETF}}^{T>0}[\rho]=\tau_{\mathrm{ETF}}^{T=0}[\rho]+\left(2 m^{*} / \hbar^{2}\right) \alpha(\rho) T^{2}, \\
& \sigma_{\mathrm{TF}}[\rho]=2 \alpha(\rho) T,
\end{aligned}
$$

where

$\alpha(\rho)=\frac{1}{12}\left(3 \pi^{2}\right)^{1 / 3}\left(2 m^{*} / \hbar^{2}\right) \rho^{1 / 3}(r)$,

and $m^{*}$ is a constant effective nucleon mass. Note that the spatial integral of $\alpha(\rho)$ eq. (4) is nothing but the TF approximation to the level density parameter $a_{0}=\pi^{2} \widetilde{g}(\lambda) / 6$, where $\widetilde{g}(\lambda)$ is the average single-particle level density at the Fermi energy. The approximation eq. (3) has been used by several authors to discuss thermal properties of nuclei [19]. The low-T expansion is, however, not justified locally in the nuclear surface where $T \sim(\lambda-V)$ even at low temperatures. It is therefore not surprising that this approximation gives bad results, as was shown e.g. for the level density parameter [18] and will also be demonstrated below.

The extension of the partial resummation method $[8-10]$ to the $T>0$ case is straight forward. Here one uses the fact that the single-particle density matrix $\rho\left(r, r^{\prime}\right)$ can be expressed through an inverse Laplace transform of the Bloch density $C\left(r, r^{\prime} ; \beta\right)$ which is defined in the $T=0$ case in terms of the single-particle (HF) wavefunctions $\varphi_{i}(r)$ and energies $\epsilon_{i}$ by

$C_{0}\left(r, r^{\prime} ; \beta\right)=\sum_{i} \varphi_{i}^{*}\left(r^{\prime}\right) \varphi_{i}(r) \exp \left(-\beta \epsilon_{i}\right)$.

(Note that $\beta$ here is not an inverse temperture, but a purely mathematical variable.) Using suitable partial resummations of the semiclassical $\hbar$-expansion of $C_{0}$ and performing the Laplace inversion with the saddlepoint method (see ref. [9] for the technical details), one obtains a $\rho\left(r, r^{\prime}\right)$ and from it smooth densities $\rho(r)$ and $\tau(r)$ which are well defined also beyond the classical turning points and can be used in an iterative scheme to yield self-consistent average ground-state properties from a given effective interaction [10]. 
The generalization of eq. (5) to $T \geqslant 0$ is given by

$C\left(r, r^{\prime} ; \beta\right)=C_{0}\left(r, r^{\prime} ; \beta\right) \pi \beta T / \sin (\pi \beta T)$.

The factor multiplying the "cold" Bloch density $C_{0}$ in eq. (6) is nothing but the (two-sited) Laplace transform of the smoothing function $f_{\mathrm{T}}(E)=\frac{1}{4} \cosh ^{-2}(E / 2 T)$ with which the $(T=0)$ single-particle spectral density must be convoluted to include the finite temperature occupation numbers (see ref. [14]). Since the Laplace inversion is made numerically in the partial resummation method, the exact $T$-dependent factor in eq. (6) can be included without any complications.

In the following we shall compare some results obtained with these two semiclassical methods to those of microscopical HF calculations which have been performed earlier [20]. Hereby the Skyrme force SIII [21] was used. As in all similar calculations [20,22,23], the parameters of the force were supposed not to depend on the temperature. In the semiclassical calculations, the spherical nucleon densities were parametrized by generalized Fermi functions and their parameters determined variationally. (See refs. $[7,10]$ for the technical details.)

In fig. 1 we show the "effective level density parameter" $a_{\text {eff }}=\frac{1}{4} \mathrm{~d} S^{2} / \mathrm{d} E^{*}$ plotted versus the excitation energy $E^{*}=E(T)-E(0)$. In the Fermi gas theory, $a_{\text {eff }}$ is equal to $a_{0}$. This value is reached by the HF result [20] when the shell effects are washed out for $T \geq 2.5-3 \mathrm{MeV}$. The value obtained with the low- $T$ expanded ETF functional is more than $30 \%$ too high; a well known failure of this approximation [18]. The

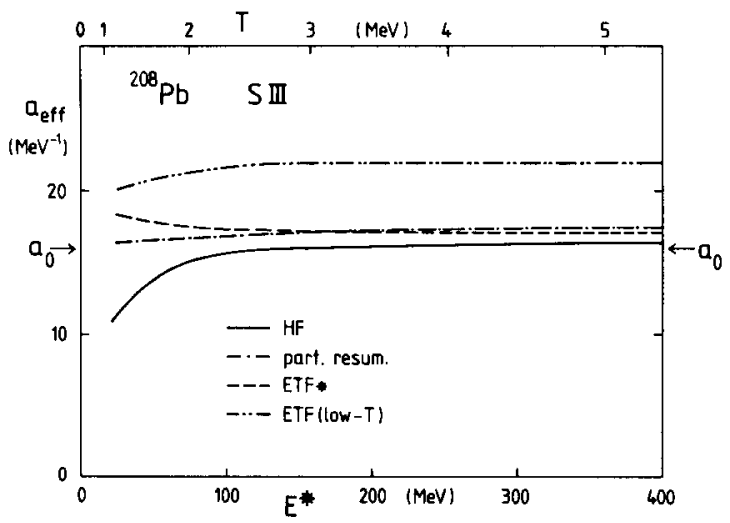

Fig. 1. Effective level density parameter $a_{\text {eff }}$ (see text) versus excitation energy $E^{*}$ for the ${ }^{208} \mathrm{~Pb}$ nucleus, obtained with the SIII Skyrme force in various approximations.

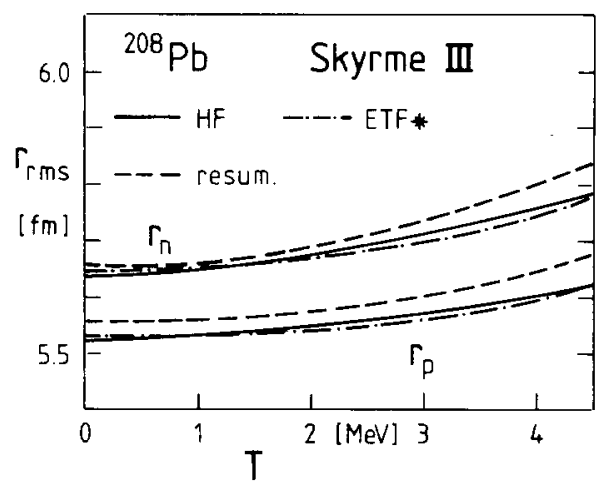

Fig. 2. Neutron and Proton RMS radii versus temperature $T$ (same force and nucleus as fig. 1).

modified functional (ETF*) eq. (2) as well as the partial resummation method lead to an asymptotic value of $a_{\text {eff }}$ only $\sim 5-8 \%$ higher than the HF result.

In fig. 2 we show the neutron and proton RMS radii of $208 \mathrm{~Pb}$ as functions of the temperature. Both our semiclassical methods are seen to reproduce the HF radii at all temperatures within less than $1 \%$. The low- $T$ expanded functional eq. (3) leads to radii (not shown in the figure) which follow the ETF* ones up to $T \simeq 2.5 \mathrm{MeV}$ but then increase much too steeply.

We want to emphasize that the agreement found in these results also signify a confirmation of the ad hoc ansat $z$ for the ETF* functional eq. (2) by the partial resummation method in which the temperature dependence is treated exactly.

We have thus two semiclassical methods at hand which give a good agreement with HF results also at finite temperature. We have not carried the above calculations for finite nuclei to higher temperatures, because at $T \gtrsim 3-4 \mathrm{MeV}$ the nucleus starts to evaporate nucleons. In more recent HF calculations in finite cells $[22,23]$, it was shown indeed, that the nucleon densities go to constant nonzero values outside the nucleus, thus forming an external gas. The effects of this external gas can, however, be practically neglected up to $T \simeq 3-4 \mathrm{MeV}$, which justifies the above calculations where the densities were always going to zero out side the nucleus.

In the following we shall apply the ETF functional method to an idealized equilibrium situation where condensed nuclear matter coexists with a gas of nucleons (and leptons) at finite temperature and finite pressure, such as it is believed to occur locally in a 
masive star during its gravitational collapse into a supernova. For the possible transition of a phase of nuclei immersed in the gas to a phase of gas bubbles in condensed nuclear matter, it was shown recently in a liquid drop model (LDM) treatment that not only the exact values of the surface and Coulomb energies but also that of the curvature energy can crucially influence the critical density [3]. It is therefore of importance to derive these LDM parameters and their temperature dependence consistently from a realistic effective interaction. The semiclassical methods discussed here provide an ideal tool for such a calculation.

The definitions at $T=0$ of the parameters of the LDM and its extension to asymmetric compressible nuclei, i.e. the droplet model, are well known [24] . Their systematic derivation from the ETF model using Skyrme forces is given in ref. [7]. For a plane interface between condensed nuclear matter and a nucleon gas at finite temperature $T$, the surface energy was recently discussed by Ravenhall et al. [17]. We shall confine ourselves here to the symmetric case without Coulomb interaction. The surface tension $\Omega_{\mathrm{s}}$ then is

$\Omega_{\mathrm{s}}=\int_{-\infty}^{+\infty}\left\{\mathcal{F}[\rho(z)]-\lambda \rho(z)+P_{0}\right\}$,

where $\mathcal{F}[\rho]$ is the free energy density functional which we obtain here for a given Skyrme force with the ETF* functional discussed above; $\lambda$ is the chemical potential and $P_{0}$ the equilibrium pressure. The "semi-infinite" density profile $\rho(z)$ is determined by minimizing $\Omega_{\mathrm{s}}$ with the boundary conditions that $\rho(z)$ approaches asymptotically the limits $\rho_{0}$ (condensed matter) and $\rho_{\mathrm{g}}$ (gas) on either side of the interface. The values $\rho_{0}, \rho_{\mathrm{g}}, \lambda$ and $P_{0}$ are found at each temperature by the well-known Maxwell construction.

Similarly as in our calculations for finite nuclei, we parametrize here $\rho(z)$ by a Fermi function and minimize $\Omega_{\mathrm{s}}$ with respect to the diffuseness parameter. Although this seems to be a rather limited variation, it gives a very good estimate of the surface energy. Indeed, it was shown in ref. [7] at $T=0$ that using more elaborate trial densities $\rho(z)$ with up to three variational parameters, the surface energies are lowered by less than $2 \%$ and agree with available HF results within $\sim 3 \%$.
Once the optimal profile $\rho(z)$ is known, the free surface energy is given by $a_{s}=4 \pi r_{0}^{2} \Omega_{\mathrm{s}}$ with $r_{0}=$ $\left(3 / 4 \pi \rho_{0}\right)^{1 / 3}$. The asymptotic values $a_{\mathrm{c}}$ and $a_{0}$ of the following coefficients in the LDM expansion of the free energy,

$F=E-T S=a_{\mathrm{v}} A+a_{\mathrm{s}} A^{2 / 3}+a_{\mathrm{c}} A^{1 / 3}+a_{0}$,

are given essentially by the first and second moments of the integrand in eq. (7); care has to be taken with terms in the energy density containing $\Delta \rho$ and $(\Delta \rho)^{2}$, which give some extra contributions [7]. For applications to finite nuclei, one also has to take into account compression effects; these do however not change the qualitative behaviour of the results presented below.

In fig. 3 we present the parameters $a_{\mathrm{s}}^{\infty}, a_{\mathrm{c}}^{\infty}$ and $a_{0}^{\infty}$ obtained in the way outlined above. (The index " $\infty$ " is used to show that compression effects are not included.) We used hereby the Skyrme force SkM* which was recently shown to give excellent fits of binding energies and radii of stable spherical nuclei in $\mathrm{HF}$ calculations [13]; it gives at the same time realistic fission barriers for heavy nuclei [7], which are a crucial measure for the surface properties. The force

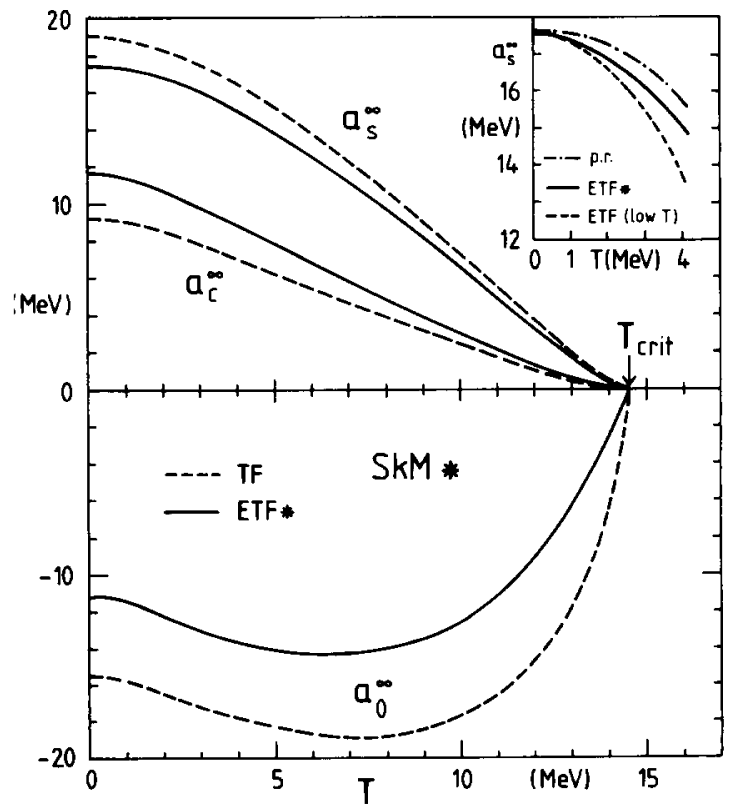

Fig. 3. Surface $\left(a_{\mathrm{s}}^{\infty}\right)$, curvature $\left(a_{\mathrm{c}}^{\infty}\right)$ and constant term $\left(a_{0}^{\infty}\right)$ of the LDM expansion of the free energy versus temperature $T$. In the ETF* approximation, spin -orbit and effective mass corrections are included. The Skyrme force SkM* was used. 
SkM* also reproduces the experimental peak energies of the giant monopole resonance (the breathing mode) throughout the mass table [25] and thus leads to a realistic compressibility. All three LDM parameters shown in fig. 3 go to zero at the critical temperature $T_{\mathrm{c}} \simeq 14.6 \mathrm{MeV}$. The other critical parameters are $\rho_{\mathrm{c}}$ $\simeq 0.053 \mathrm{fm}^{-3}$ and $P_{\mathrm{c}} \simeq 0.21 \mathrm{MeV} \mathrm{fm}^{-3}$. We also have shown in fig. 3 the results obtained using the pure TF functionals. Some TF calculations for the surface energy were presented in ref. [17]; they were then scaled upwards by $\sim 20 \%$ such as to fit the HF value of $a_{\mathrm{s}}$ at $T=0$. We can see from our results that such a scaling procedure might be used, indeed, for the surface energy alone; it would however lead to wrong values of $a_{\mathrm{c}}^{\infty}$ and $a_{0}^{\infty}$. This demonstrates that the inclusion of semiclassical corrections beyond the TF approximation is compulsory to obtain correct values of all LDM parameters.

In the insert on the upper right of fig. 3 we show a comparison of the surface energies obtained with the three above approximations in the region $0 \leqslant T \leqslant 4$ $\mathrm{MeV}$ where the results are unchanged if one puts $\rho_{\mathrm{g}}=$ $P_{0}=0$. Clearly, the low $-T$ expanded ETF functional leads again to an overestimation of the finite temperature effects, as compared to the ETF* functional. The partial resummation method leads to a still slower decrease of $a_{\mathrm{s}}^{\infty}$ with $T$. This is partially due to numerical effects because with this method, the surface energy $a_{s}^{\infty}$ was here not calculated from the semi-infinite geometry, but by a graphical extraction from the free energies $F$ of large finite nuclei with $N=Z$. Although this procedure in principle leads to the same value of $a_{\mathrm{s}}^{\infty}$, it becomes numerically less reliable with increasing $T$.

We shall not discuss here the asymmetry energy parameters of the LDM. (See ref. [7] for a droplet model analysis of ETF results at $T=0$.) The force $\mathrm{SkM} *$ is known not to reproduce too well the binding energies of nuclei far off the $\beta$-stability line, and has therefore little predictive power for asymmetry parameters. Nevertheless, we can mention here the schematic result that the (free) surface asymmetry energy (which is $\sim-60 \mathrm{MeV}$ at $T=0$ ) is found to increase with temperature in its absolute value, which is the result of a competition between the variation of the volume asymmetry energy $J$ and that of the surface stiffness parameter $Q$ of the droplet model [24]. (For the SkM* force we obtain $J=30 \mathrm{MeV}$ and $Q=35.4 \mathrm{MeV}$ at $T=0$.)
The calculation of asymmetry energies from improved Skyrme forces and, more generally, the discussion of a temperature-dependent droplet model including com. pression effects, will be the subjects of further publications.

In summary, we have shown that semiclassical methods can quantitatively replace the much more complicated HF calculations for highly excited nuclear systems and are an ideal tool for the calculation of LDM parameters from an effective interaction. We have also demonstrated that the semiclassical corrections beyond the TF approximation play an important role. In closing we mention that the ETF* functional has been used also to study the temperature dependence of fission barriers [7] and of monopole and dipole sum rules [26].

We are grateful to J. Meyer and W. Stocker for encouraging discussions. One of us (M. Brack) acknowledges the hospitality of the Département de Recherche Fondamentale at the CENG in Grenoble during a sabbatical visit, where part of the calculations were done.

\section{References}

[1] See, e.g., M. Lefort, Nucl. Phys. A387 (1982) 3c.

[2] See, e.g., J.M. Lattimer, Ann. Rev. Nucl. Part. Sci. 31 (1981) 337.

[3] C.J. Pethick, D.G. Ravenhall and J.M. Lattimer, Phys. Lett. 128B (1983) 137.

[4] W. Stocker and M. Farine, Preprint (1983), and private communication.

[5] D.A. Kirzhnits, Field theoretical methods in many body systems (Pergamon, Oxford, 1967).

[6] M. Brack, B.K. Jennings and Y.H. Chu, Phys. Lett. 65 B (1976) 1;

B. Grammaticos and A. Voros, Ann. Phys. 123 (1979) 359; 129 (1980) 153.

[7] M. Brack, C. Guet and H.-B.Håkansson, to be submitted to Phys. Rep.

[8] R.K. Bhaduri, Phys. Rev. Lett. 39 (1977) 329; M. Durand, M. Brack and P. Schuck, Z. Phys. A 286 (1978) 381 .

[9] J. Bartel, M. Durand and M. Brack, Z. Phys. A, to be published.

[10] J. Bartel and M. Vallières, Phys. Lett. 114 B (1982) 303; J. Bartel, to be published.

[11] C. Guet and M. Brack, Z. Phys. A 297 (1980) 247.

[12] C. Guet, H.-B. Hảkansson and M. Brack, Phys. Lett. 97 B (1980) 7.

[13] J. Bartel et al., Nucl. Phys. A386 (1982) 79.

[14] M. Brack and P. Quentin, Nucl. Phys. A361 (1981) 35. 
[15] W.A. Küpper, G. Wegmann and E. Hilf, Ann. Phys. 88 (1974) 454;

M. Barranco and J.R. Buchler, Phys. Rev. C 22 (1980) 1729 ;

D.Q. Lamb et al., Nucl. Phys. A 360 (1981) 459

[16] W. Stocker and J. Burzlaff, Nucl. Phys. A 202 (1973) 265.

[17] D.G. Ravenhall, C.J. Pethick and J.M. Lattimer, Nucl. Phys. A 407 (1983) 571.

[18] M. Barranco and J. Treiner, Nucl. Phys. A 351 (1981) 269.

[19] M. Barranco, M. Pi and X. Viñas, Phys. Lett. 124 B (1983) 131;

X. Campi and S. Stringari, Z. Phys. A 309 (1983) 239.
[20] M. Brack and P. Quentin, Phys. Scr. 10 A (1974) 163; Phys, Lett. 52 B (1974) 159.

[21] M. Beiner et al., Nucl. Phys. A 238 (1975) 433.

[22] P. Bonche and D. Vautherin, Nucl. Phys. A 372 (1981) 496.

[23] P. Bonche, S. Levit and D. Vautherin, Saclay-Preprint $\mathrm{SPhT} / 83 / 85$.

[24] W.D. Myers and W.J. Swiatecki, Ann. Phys. 55 (1969) 395.

[25] M. Brack and W. Stocker, Nucl. Phys. A 406 (1983) 413.

[26] J. Meyer, P. Quentin and M. Brack, Phys. Lett. 133 B (1983) 279. 